\title{
Modelo 3-3-1 con neutrinos derechos, implementado en SARAH y SPheno
}

\author{
A. Tapia ${ }^{a}$, and R. Benavides ${ }^{b}$ \\ ${ }^{a}$ Universidad de Medellín, Carrera $87 N^{\circ} 30$ - 65, Medellín, Colombia. \\ ${ }^{b}$ Instituto Tecnológico Metropolitano, Calle $73 N^{o} 76$ A-354, Vía el Volador, Medellín Colombia.
}

Received 6 August 2020; accepted 4 September 2020

\begin{abstract}
En este trabajo se presenta por primera vez la implementación del modelo 3-3-1 con neutrinos derechos sin cargas eléctricas exóticas en el paquete SARAH del programa MATHEMATICA y se muestra cómo reproduce de manera correcta los resultados analíticos del modelo. Como prueba de ello, se presentan las matrices de masa para los sectores de quarks donde se ha usado un sector de Higgs con tres tripletes escalares. Luego, usando el programa SPheno, se realiza un análisis numérico de las salidas analíticas obtenidas con SARAH con el objetivo de determinar si el modelo con tres tripletes genera los valores de masas de todos los quarks adecuadamente al compararlos con los aceptados en la literatura. Esta implementación se presenta de manera didáctica y accesible a estudiantes interesados en este campo de la física.
\end{abstract}

Descriptores: Modelo 3-3-1; SARAH; SPheno.

In this work, for the first time, the implementation of the 3-3-1 model with right-handed neutrinos without exotic electric charges in the SARAH package of MATHEMATICA is presented, and it shows how it correctly reproduces the analytical results of the model.As proof of this, we present the mass matrices for the quark sectors, where a Higgs sector with three scalar triplets has been used. Then, using the SPheno program, a numerical analysis of the analytical outputs obtained with SARAH is performed; this with the objective of determining if the model with three triplets generates the mass values of all quarks adequately when comparing them with those accepted in the literature. This implementation is presented in a didactic and accessible way to students who are interested in this field of physics.

Keywords: 3-3-1 model; SARAH; SPheno.

PACS: $98.80 \mathrm{Cq} ; 03.70+\mathrm{k} ; 12.20-\mathrm{m} ; 29.50+\mathrm{v}$

DOI: https://doi.org/10.31349/RevMexFisE.18.107

\section{Introducción}

Se conoce que el Modelo Estándar (ME) de la física de partículas no puede ser una teoría definitiva, por lo tanto se buscan modelos que expliquen algunos de los tópicos que el ME no puede explicar, siempre que dichos modelos estén de acuerdo con el ME. Por esta razón lo más simple es ampliar el ME, lo cual se logra aumentando el sector fermiónico, aumentando el sector escalar a más de una representación del Higgs, o ampliando el grupo Gauge local. En este orden de ideas, se presenta el modelo teórico basado en el grupo Gauge local $S U(3)_{c} \otimes S U(3)_{L} \otimes U(1)_{X}(3-3-1$ para acortar) [1-6].

Los resultados experimentales en el CERN-LEP realizado en los años 90 concluyen la existencia de al menos tres familias fermionicas. Los modelos $3-3-1$ explican de manera natural la razón por el cual número de familias debe ser tres al tratar el álgebra de corrientes y cancelar las anomalías que se presentan en este sector. Lo anterior se logra gracias a que, en estos modelos, las primeras dos familias tienen números cuánticos distintos a la tercera familia.

Las principales características de los modelos $3-3-1$ son:

- En algunos de estos modelos, las anomalías Gauge se cancelan solamente si el número de familias es múltiplo de 3 [5,7-9] (para garantizar la libertad asintótica de $\left.S U(3)_{c}\right)$.
- La simetría de Peccei-Quinn puede implementarse fácilmente [10,11].

- Una familia de quarks tiene números cuánticos distintos, hecho que puede explicar el valor tan grande de la masa del quark top $[4,12]$.

- El sector escalar posee buenos candidatos a materia oscura [13-15].

- El sector leptónico es capaz de describir algunas propiedades de los neutrinos [16-18].

- La jerarquía en las constantes de acoples de Yukawa puede evitarse al implementar el mecanismo "see-saw" universal [4, 19-22].

Lo anterior genera un gran atractivo para el estudio de estos modelos. Se asume que el grupo gauge electrodébil $S U(3)_{L} \otimes U(1)_{X} \supset S U(2)_{L} \otimes U(1)_{Y}$ y que los quarks de quiralidad izquierda son tripletes de color, y los leptones de quiralidad izquierda son singletes de color, los cuales transforman bajo las dos representaciones fundamentales $\left(3\right.$ y $\left.3^{*}\right)$.

Uno de los sectores básicos de los modelos de la física de partículas es el relacionado con el sector del Higgs responsable de que los campos adquieran masa. En el marco de los modelos 3-3-1 este también debe aumentarse, por ello, se analizará el modelo con tres tripletes escalares presentado en [23].

Por otro lado, se encuentran las herramientas computacionales para obtener de manera más rápida resultados que de 
otra manera tomaría mucho tiempo resolver o que resultaría imposible hallar. Así pues, dicho desarrollo ha contribuido a grandes avances en la ciencia. En este sentido, usaremos el paquete de uso libre SARAH [24,25], el cual se ejecuta a través del programa MATHEMATICA (producto protegido por Wolfram Research) [26], para analizar uno de los modelos 3-3-1 sin cargas eléctricas exóticas (hemos escogido el modelo conocido como 3-3-1 con neutrinos derechos, descrito más adelante). Adicionalmente, se analizará el modelo usando la salida de SARAH como módulo del programa SPheno [24, 25, 27, 28], con el objetivo de realizar cálculos numéricos a nivel árbol para los quarks del modelo y mostrar si es posible tener un modelo realista o no, con tres tripletes escalares.

El artículo está organizado como sigue. En la Sec. 2 se presenta el modelo 3-3-1 con neutrinos derechos a implementar con tres tripletes escalares. En la Sec. 3 se presenta la implementación del modelo 3-3-1 en SARAH. En la Sec. 4 se muestran las salidas analíticas que se obtienen con SARAH y se comparan con algunos resultados reportados en la literatura científica. En la Sec. 5 se presentan los resultados numéricos generados con SPheno para la salida dada por SARAH y en la Sec. 6 se presentan las conclusiones. Un Apéndice al final muestra los archivos creados para lograr una implementación completa en SARAH del modelo 3-3-1 con tres tripletes de Higgs.

\section{Modelo 3-3-1 con neutrinos derechos}

Los modelos 3-3-1 han sido ampliamente estudiados dentro de la literatura científica. Se trata de modelos libres de anomalías de Gauge, renormalizables, con sector fermiónico, bosónico y escalar aumentado con respecto al Modelo Estándar. Para mayor información, el lector se puede dirigir a [1-6]. En este caso analizaremos uno de los modelos 3-3-1 sin cargas eléctricas exóticas según la clasificación presentada en [23], conocido como modelo 3-3-1 con neutrinos derechos, con tres tripletes escalares. Los campos del modelo son:

\subsection{Sector fermiónico}

El sector de quarks del modelo es: $Q_{L}^{i}=\left(u^{i}, d^{i}, D^{i}\right)_{L} \sim$ $(3,3,0), i=1,2$, para las primeras dos familias, donde $D_{L}^{i}$ se refieren a dos quarks extras de carga eléctrica $-1 / 3$, mientras que, los números cuánticos entre paréntesis se refieren a las simetrías $\left[S U(3)_{c}, S U(3)_{L}, U(1)_{X}\right], Q_{L}^{3}=$ $\left(d^{3}, u^{3}, U^{3}\right)_{L} \sim\left(3,3^{*}, 1 / 3\right)$, donde $U_{L}$ es un quark tipo up extra. Los quarks derechos son $u_{L}^{a c} \sim\left(3^{*}, 1,-2 / 3\right)$, $d_{L}^{a c} \sim\left(3^{*}, 1,1 / 3\right)$ con $a=1,2,3$ índice de familia, $D_{L}^{i c} \sim$ $\left(3^{*}, 1,1 / 3\right), i=1,2$ y $U_{L}^{c} \sim\left(3^{*}, 1,-2 / 3\right)$.

El sector leptónico es: $L_{L}^{l}=\left(l^{-}, \nu_{l}^{0}, \nu_{l}^{0 c}\right)_{L} \sim$ $\left(1,3^{*},-1 / 3\right)$, para $l=e, \mu, \tau$, para el índice de familia leptónico y los tres singletes $l_{L}^{+} \sim(1,1,1)$ donde $\nu_{l}^{0}$ es el campo asociado al neutrino y $\nu_{l}^{0 c}$ desempeña el rol de neutrino derecho.

\subsection{Sector escalar}

En el marco de los modelos $3-3-1$ sin cargas eléctricas exóticas. El sector escalar que usaremos consta de tres tripletes escalares como se presenta en la Ref. [23]:

$$
\begin{gathered}
\Phi_{1}(1,3,1 / 3)=\left(\begin{array}{c}
\phi_{1}^{+} \\
\phi_{1}^{0} \\
\phi_{1}^{\prime 0}
\end{array}\right), \text { con VEV: }\left\langle\Phi_{1}\right\rangle=\left(\begin{array}{c}
0 \\
0 \\
V
\end{array}\right), \\
\Phi_{2}(1,3,1 / 3)=\left(\begin{array}{c}
\phi_{2}^{+} \\
\phi_{2}^{0} \\
\phi_{2}^{\prime 0}
\end{array}\right), \text { con VEV: }\left\langle\Phi_{2}\right\rangle=\left(\begin{array}{c}
0 \\
v_{1} \\
0
\end{array}\right), \\
\Phi_{3}(1,3,-2 / 3)=\left(\begin{array}{c}
\phi_{3}^{0} \\
\phi_{3}^{-} \\
\phi_{3}^{\prime \prime}
\end{array}\right), \text { con VEV: }\left\langle\Phi_{3}\right\rangle=\left(\begin{array}{c}
v_{2} \\
0 \\
0
\end{array}\right),
\end{gathered}
$$

con la jerarquía $v_{1} \sim v_{2} \sim 10^{2} \mathrm{GeV}<<V \sim \mathrm{TeV}$. El rompimiento de la simetría de $S U(3)_{c} \otimes S U(3)_{L} \otimes U(1)_{X}$ se lleva a cabo en dos etapas: el primer rompimiento es hacia el ME y el segundo hacia el electromagnetismo.

Deseamos mostrar que bajo el esquema del rompimiento de simetría de tres tripletes escalares, SARAH y SPheno funcionan, respectivamente, para el análisis analítico y para el análisis numérico de los archivos generados por SARAH. Lo anterior reduce de manera considerable el tiempo de cálculo.

Respecto al sector bosónico Gauge, es el mismo para todos los modelos 3-3-1 sin cargas eléctricas exóticas que bajo este esquema de tres tripletes escalares han sido bastante bien analizados en la literatura. Lo anterior se puede consultar en las referencias $[6,9]$.

\section{Implementación del modelo en SARAH}

Como ya se mencionó, SARAH es un paquete de uso libre que funciona bajo el programa MATHEMATICA, el cual permite implementar y analizar analíticamente modelos de física de partículas [24,25]. SARAH no requiere ninguna compilación; después de descargar el paquete, se descomprime en el directorio de aplicaciones de MATHEMATICA. Para implementar un nuevo modelo, el primer paso consiste en es crear un nuevo directorio en \$PATH/SARAH-X.Y.Z/Models con el nombre del nuevo modelo. Dentro de este directorio se deben generar cuatro archivos. El primero es el archivo principal, que lleva el nombre del nuevo modelo. Para nuestro caso será 331-v3.m. En este se definen los multipletes del modelo, el lagrangiano del mismo, las simetrías de Gauge y la manera como se romperán. Luego se genera el archivo parameters.m, que provee información adicional acerca de los parámetros del modelo. También se debe generar el archivo particles.m, aquí se definen las partículas del modelo, con algunos detalles no presentes en $331-\mathrm{v} 3 . \mathrm{m}$. Finalmente, se debe crear el archivo SPheno.m; este se requiereúnicamente si se desea crear un módulo de SPheno para realizar cálculos numéricos con el modelo. Estos cuatro archivos se presentan a detalle en el Apéndice A. Para 
mayor información sobre la implementación de un modelo en SARAH, consultar las Refs. [24,25].

El modelo a trabajar posee la estructura Gauge $S U(3)_{c} \otimes$ $S U(3)_{L} \otimes U(1)_{X}$, la cual se define dentro del archivo principal 331-v3.m de SARAH en la forma:

$$
\begin{array}{lll}
\text { Gauge }[[1]]=\{B, \quad U[1], & \text { xcharge, } & \text { g1, False }\} ; \\
\text { Gauge }[[2]]=\{W B, S U[3], & \text { left, } & \text { g2, True }\} ; \\
\text { Gauge }[[3]]=\{G, S U[3], & \text { color, } & \text { g3, False }\} ;
\end{array}
$$

El primer elemento hace referencia al nombre del bosón de Gauge; el siguiente es el grupo Gauge, el nombre del grupo, el nombre de la constante de acoplamiento, y True o False. Esto es relevante si la simetría se rompe como en el caso de de $S U(3)_{L}$ y existe un índice que corre para los generadores del grupo.

El siguiente paso es introducir uno a uno los campos fermiónicos y los campos escalares de la siguiente manera:

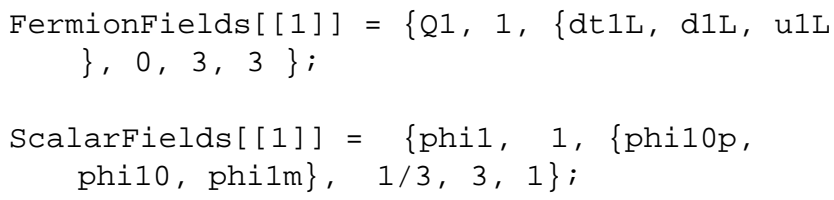

donde la primera entrada corresponde al nombre del campo, la segunda es el número de generaciones y el tercero es el nombre de las componentes de $S U(3)_{L}$. La demás entradas son las cargas bajo el Gauge 3-3-1.

También se debe definir el lagrangiano completo del modelo y la mezcla entre los bosones de Gauge, tanto entre los neutros como para los cargados:

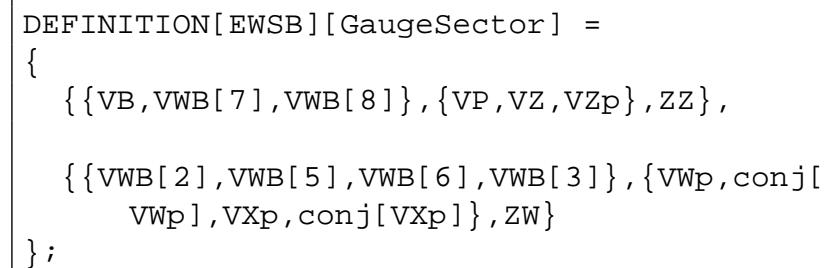

En la primera línea se definen los bosones de Gauge neutros, $\left\{B, W_{7}, W_{8}\right\} \rightarrow\left\{\gamma, Z, Z^{\prime}\right\}$, donde $\mathrm{ZZ}$ es la matriz de mezcla, mientras que en la siguiente línea se definen los bosones de Gauge cargados, $\left\{W_{2}, W_{5}, W_{6}, W_{3}\right\} \rightarrow$ $\left\{W^{+}, W^{-}, W^{\prime+}, W^{\prime-}\right\}$, donde $\mathrm{zW}$ es la matriz de mezcla para este caso.

Lo siguiente es definir los Higgs que adquieren valor de expectación en el vacío, así como también los componentes cp-par y cp-impar del sector escalar. Después se definen los estados de masa para los fermiones, tanto las matrices de masa como las masas de Majorana o masas de Dirac; esto se logra definiendo los estados espinoriales de los mismos, ya que SARAH considera a todos los fermiones como estados de Weyl de dos componentes. Por esta razón es importante clarificar en SARAH cómo se deben combinar a través de cuatro estados de Weyl para generar un estado de Dirac, si fuese el caso. La implementación completa de todo lo anterior se muestra en la primera sección del Apéndice A.

El siguiente archivo que se debe generar en SARAH es el parameters.m. En este archivo se debe introducir información extra del modelo, para definir el nombre de las matrices de mezcla, los ángulos de mezcla, y cada una de las constantes de acoplamiento, con sus respectivos nombres de salida hacia otros programas como, $\mathrm{LT}_{\mathrm{E}} \mathrm{X}$, SPheno y hasta MicrOmegas, en caso de querer analizar materia oscura del modelo. En esta parte es posible definir ciertos parámetros en función de valores bien conocidos dentro del ME, como por ejemplo, el ángulo de Weinberg, la constante de estructura fina, la carga del electrón, etc...

Finalmente, el último archivo antes de completar el modelo implementado en SARAH y así ejecutarlo para obtener las salidas analíticas en MATHEMATICA es el archivo particles.m. Este archivo está relacionado con las partículas del modelo. Aunque la información básica ya se ingresó en el archivo principal, hay algunos detalles adicionales que se deben incluir en este archivo. Así como parameters.m, este archivo es opcional pero se recomienda implementarlos cuando el modelo cuenta con parámetros y partículas poco comunes que SARAH no pueda identificar causando un error, además cuando se requiera generar los archivos especiales de salida de SARAH para SPheno, $\mathrm{LT}_{\mathrm{E}} \mathrm{X}$, etc. En particles.m, por ejemplo, se ingresa la carga de las partículas, se define el valor numérico (en caso de ser conocido) de las masas de algunas partículas, o se define la manera en que los programas externos pueden leer sus valores usando un archivo de entrada generado por SPheno. También está la opción de presentar cómo las partículas deberían mostrarse en el formato $\mathrm{HTEX}_{\mathrm{E}}[24,25]$.

\section{Resultados Analíticos}

Una vez implementado el modelo, primero se debe cargar el paquete SARAH desde MATHEMATICA ejecutando la siguiente orden:

\section{$<$ /home/.Mathematica/Applications/SARAH-X.Y . Z/SARAH.m}

donde X.Y. Z indica los tres dígitos de la versión usada para SARAH. Esta orden carga el paquete y despliega información sobre SARAH: 


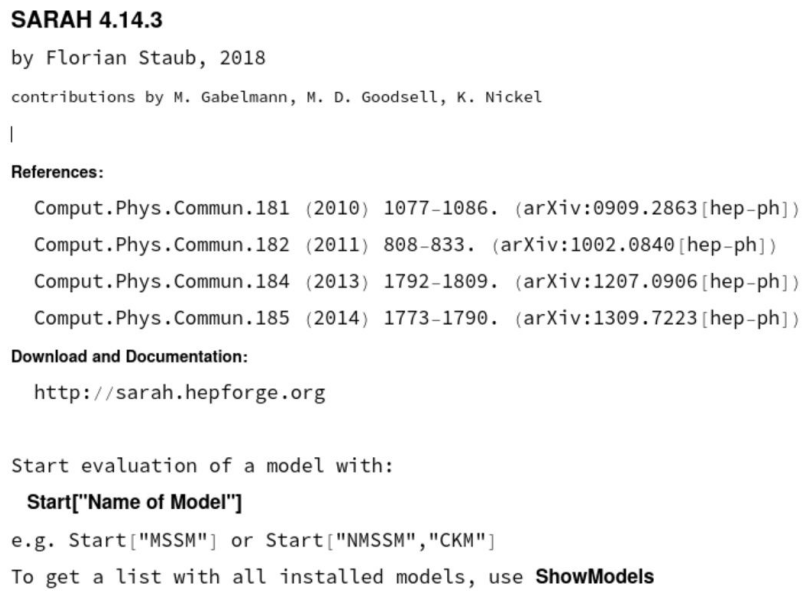

Al final se muestra la forma en que debe iniciar el modelo implementado; para nuestro caso debemos ejecutar

| Start ["331/v3"]

SARAH empieza verificando que el modelo cumpla con los requerimientos básicos, como preservar la carga en cada término del modelo y que el sector de Gauge esté adecuadamente construido, es decir, se deben especificar correctamente los bosones de Goldstone que representarán los grados de libertad que requieran los bosones masivos para adquirir masa Asimismo, se debe verificar que la mezcla y los pseudoescalares en el sector de Higgs se encuentren bien definidos. Después de ello, el programa encontrará todos los vértices del modelo, las matrices de masa, las ecuaciones Tadpoles y en caso de ser necesario, también encuentra correcciones a 1-loop para las Tadpoles, las auto-energías y hasta 2-loops para las ecuaciones del grupo de renormalización.

Como resultado de la ejecución se obtienen las simetrías de Gauge, todos los campos del modelo, el Lagrangiano completamente expandido, las matrices de mezcla de los bosones de Gauge, las matrices de rotación de masa tanto para los bosones de Gauge como para los campos escalares del modelo, neutros y cargados, las matrices de masa para quarks y leptones una vez que hayan interactuado con el sector del Higgs. Inclusive se tiene la opción de exportar los resultados analíticos a archivos LTEX para obtener un archivo PDF como resultado de una compilación, el cual incluya todos los detalles del modelo, en donde hasta los vértices son graficados después de usar el programa de LTEX-feynmf. Todo esto muestra que SARAH es una herramienta muy completa para realizar análisis de modelos en física de altas energías.

Parte de nuestro interés es mostrar las matrices de masa del sector de quarks, y como caso siguiente, determinar a través de SPheno los valores numéricos de las masas de dichos quarks. Por lo tanto, a continuación mostramos las matrices de masa analíticas que se obtienen al ejecutar el programa en SARAH con tres tripletes escalares.

Para obtener la matriz de masa del sector Up de quarks se debe ejecutar el comando:

| MatrixForm [MassMatrix [Fu] ] y SARAH retorna la forma conocida de la matriz de masa,

$\left(\begin{array}{cccc}\text { v2 hup11 [1] } & \text { v2 hup11[2] } & \text { v2 hup11 [3] } & \text { hUp1 v2 } \\ \text { v2 hup21[1] } & \text { v2 hup21[2] } & \text { v2 hup21[3] } & \text { hUp2 v2 } \\ \text { v1 hu12[1] } & \text { v1 hu12[2] } & \text { v1 hu12[3] } & \text { hU2 v1 } \\ \text { v hu11[1] } & \text { v hu11[2] } & \text { v hu11[3] } & \text { hU1 v }\end{array}\right)$

Para poder compararla con la publicada en la literatura, esta matriz se puede reescribir en la forma:

$$
M_{u}=\left(\begin{array}{llll}
v_{2} h_{11}^{u^{\prime}} & v_{2} h_{12}^{u^{\prime}} & v_{2} h_{13}^{u^{\prime}} & v_{2} h_{1}^{U^{\prime}} \\
v_{2} h_{21}^{u^{\prime}} & v_{2} h_{22}^{u^{\prime}} & v_{2} h_{23}^{u^{\prime}} & v_{2} h_{2}^{U^{\prime}} \\
v_{1} h_{12}^{u} & v_{1} h_{22}^{u} & v_{1} h_{32}^{u} & v_{1} h_{2}^{U} \\
V h_{11}^{u} & V h_{21}^{u} & V h_{31}^{u} & V h_{1}^{U}
\end{array}\right)
$$

Ahora para el sector Down de quarks se debe ejecutar el mismo comando, pero cambiando el estado $\mathrm{Fu}$ por $\mathrm{Fd}$, es decir:

\section{MatrixForm [MassMatrix [Fd] ]}

y SARAH nuevamente retorna la forma conocida de la matriz de masa para este sector,

\begin{tabular}{|c|c|c|c|c|}
\hline v1 hd1 [1] & v1 hd1 [2] & v1 hd1 [3] & v1 hdt 1 [1] & [2] \\
\hline v1 hd2 [1] & v1 hd2 [2] & v1 hd2 [3] & v1 hdt $2[1]$ & v1 hdt2 [2] \\
\hline v2 hd3 [1] & v2 hd3 [2] & v2 hd3 [3] & $v 2 \operatorname{hdt} 3[1]$ & $\mathrm{v} 2 \mathrm{hdt} 3[2]$ \\
\hline V hdp11 [1] & V hdp 11 [2] & V hdp 11 [3] & V hdtp11 [1] & V hdtp11 [2] \\
\hline V hdp1 [1] & V hdp1 [2] & V hdp1 [3] & V hdtp1 [1] & V hdtp1 [2] \\
\hline
\end{tabular}

Al igual que la anterior matriz, la reescribimos para compararla con la publicada en la literatura:

$$
M_{d}=\left(\begin{array}{lllll}
v_{1} h_{11}^{d} & v_{1} h_{12}^{d} & v_{1} h_{13}^{d} & v_{1} h_{11}^{D} & v_{1} h_{12}^{D} \\
v_{1} h_{21}^{d} & v_{1} h_{22}^{d} & v_{1} h_{23}^{d} & v_{1} h_{21}^{D} & v_{1} h_{22}^{D} \\
v_{2} h_{31}^{d} & v_{2} h_{32}^{d} & v_{2} h_{33}^{d} & v_{2} h_{31}^{D} & v_{2} h_{32}^{D} \\
V h_{11}^{d^{\prime}} & V h_{12}^{d^{\prime}} & V h_{13}^{d^{\prime}} & V h_{11}^{D^{\prime}} & v_{2} h_{12}^{D^{\prime}} \\
V h_{1}^{d^{\prime}} & V h_{2}^{d^{\prime}} & V h_{3}^{d^{\prime}} & V h_{1}^{D^{\prime}} & V h_{2}^{D^{\prime}}
\end{array}\right) .
$$

Si estas matrices se comparan con las presentadas en la Ref. [23], salvo algún cambio de notación en los subíndices de las constantes de Yukawa, son iguales. En este trabajo evitamos usar una mayor cantidad de subíndices para facilitar la implementación del lagrangiano del modelo en SARAH, por ejemplo, para el sector Down, en lugar de escribir $h_{211}^{d}$ usamos $h_{1}^{d^{\prime}}$. Lo importante es verificar que las constantes de Yukawa sean diferentes una respecto a la otra. Al considerar lo anterior, se muestra que la implementación en SARAH concuerda.

Ya el lector puede indagar en las diferentes salidas de SARAH, como las matrices de masa de los leptones, las matrices para los escalares cargados y neutros, las matrices de los bosones de Gauge, etc. Para mayor información de cómo obtener más salidas, remitirse a [24,25], donde encontrará toda la información que se puede extraer desde SARAH. Vale la pena recordar que por primera vez se realiza la implementación de este modelo en SARAH y que la presentamos completamente en el Apéndice A. 


\section{Resultados Numéricos usando SPheno}

Al tener certeza de que los resultados analíticos obtenidos con SARAH concuerdan con los publicados en la literatura, se procede a realizar cálculos numéricos usando el programa SPheno, con el objetivo de obtener las masas del sector de quarks para el caso con tres tripletes de Higgs. Estos cálculos numéricos se realizan a nivel árbol, la cual representa una de los opciones dadas por el programa de SPheno.

Al ser SARAH un paquete de MATHEMATICA, no es lo más indicado para realizar estudios numéricos pesados [25]. No obstante, se utiliza para generar los archivos de entrada (que llamaremos módulo) requeridos para ejecutar SPheno. SPheno es un programa escrito en lenguaje Fortran, el cual provee de rutinas que permiten la evaluación numérica de todos los vértices, masas y modos de decaimiento en un modelo determinado.

Para crear un módulo de SPheno con SARAH se necesitan hacer dos cosas: (1) crear el archivo SPheno.m (ya mencionado en la Sec. 4 y mostrado en detalle en el Apéndice 6 para nuestro modelo 3-3-1 con tres tripletes escalares) y (2) ejecutar el siguiente comando en MATHEMATICA después de cargar SARAH e inicializar el modelo:

|MakeSPheno[ ]

Después de unos minutos, el módulo de SPheno será creado y localizado en el directorio Output de SARAH. Una vez creado, se debe copiar en el directorio principal de SPheno y luego se necesita compilar, escribiendo en un terminal:

| \$make Model=331v3

y esperar hasta que la compilación termine con éxito. Cuando esto suceda, ya queda listo para usar nuestro código de SPheno ejecutando:

|\$bin/SPheno331v3 LesHouches.in.331v3_low,

donde LesHouches es el archivo que contiene los valores numéricos de entrada para las constantes físicas del modelo.

\begin{tabular}{|c|c|}
\hline Block MODSEL & \# \\
\hline 10 & 1/0: High/low scale \\
\hline input & \\
\hline 21 & \# Boundary Condition \\
\hline 61 & \# Generation Mixing \\
\hline 12173.5 & \# Renormalization scale \\
\hline Block SMINPUTS & \# Standard Model inputs \\
\hline $21.166370 \mathbf{E}-05$ & \# G_F,Fermi constant \\
\hline $31.187000 \mathbf{E}-01$ & \# alpha_s (MZ) SM MSbar \\
\hline $49.118870 \mathbf{E}+01$ & \# Z-boson pole mass \\
\hline $54.180000 \mathbf{E}+00$ & \# m_b (mb) SM MSbar \\
\hline $61.735000 \mathbf{E}+02$ & \# m_top (pole) \\
\hline $71.776690 \mathbf{E}+00$ & $\#$ m_tau (pole) \\
\hline Block MINPAR & \# Input parameters \\
\hline $115.00 \mathbf{E}+03$ & \# VnIN \\
\hline $121.00 \mathbf{E}+02$ & \# $\mathrm{v} 1 \mathrm{IN}$ \\
\hline $132.45 \mathbf{E}+02$ & $\#$ v2IN \\
\hline
\end{tabular}

Por ejemplo, en este archivo se especifican los valores de expectación en el vacío, para nuestro caso tenemos: $v_{1}=$
$100, v_{2}=245, \mathrm{y} V=5000 \mathrm{GeV}$. El valor de $v_{1}$ se puede mover desde $1 \mathrm{GeV}$ hasta $v_{1} \approx v_{2}$, y $V$ dentro de la escala de los $\mathrm{TeV}$, para garantizar el valor de las masas de los valores de las partículas exóticas.

Con los valores de expectación mencionados anteriormente se obtiene la solución más simple para el sector Up de los quarks:

$M_{u p}=h_{11}^{u^{\prime}} v_{2}, M_{c}=h_{22}^{u^{\prime}} v_{2}, M_{t}=h_{32}^{u} v_{1}$ y $M_{U}=h_{1}^{U} V$, con todas las otras constantes de Yukawa de la matriz de la Ec. (2) iguales a cero. Al fijar en el archivo de entrada LesHouches del módulo de SPheno las constantes de Yukawa en los siguientes valores:

$h_{11}^{u^{\prime}}=9.35 \times 10^{-6}, h_{22}^{u^{\prime}}=0.7053, h_{32}^{u}=0.01275, h_{1}^{U}=1$, se obtienen de manera adecuada los valores de masa de los quarks reportados en el Particle Data Group (PDG) [29], y un valor de masa para el quark exótico de $5 \mathrm{TeV}$, el cual fácilmente se puede modificar al cambiar el valor de la constante $h_{1}^{U}$.

Si se produce de la misma manera para el sector down se tiene:

$M_{d}=h_{11}^{d} v_{1}, M_{s}=h_{22}^{d} v_{1}, M_{b}=h_{33}^{d} v_{2}, M_{D 1}=h_{11}^{D^{\prime}} V \mathrm{y}$ $M_{D 2}=h_{2}^{D^{\prime}} V$;

donde las otras constantes de Yukawa de la matriz de la Ec. (3) son iguales a cero. Nuevamente, al fijar en el archivo de entrada LesHouches del módulo de SPheno las constantes de Yukawa en los siguientes valores:

$h_{11}^{d}=0.000048, h_{22}^{d}=0.00095, h_{33}^{d}=0.016992, h_{11}^{D^{\prime}}=$ 0.3 y $h_{2}^{D^{\prime}}=0.6$,

se obtienen de manera adecuada los valores de masa de los tres quarks tipo down reportadas en el PDG y los valores de masa de $1.5 \mathrm{TeV}$ y $3 \mathrm{TeV}$ para los quarks exóticos, respectivamente. Estos últimos valores fácilmente se pueden variar al cambiar los valores de $h_{11}^{D^{\prime}}$ y $h_{2}^{D^{\prime}}$. La selección de las constantes de Yukawa anterioremente mencionadas es una posibilidad de muchas que se podrían elegir en las matrices (2) y (3).

Los resultados numéricos calculados por SPheno son guardados en múltiples archivos generados en el directorio principal. Los anteriores valores correspondientes a la masas son guardados en el archivo llamado SPheno.spc.331v3. Este contiene todos los detalles del espectro de masas del modelo:

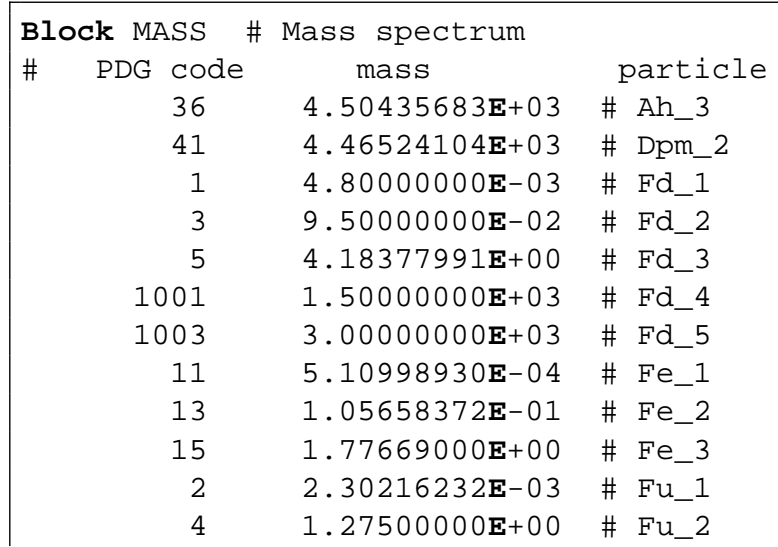




\begin{tabular}{rll||}
6 & $1.73659367 \mathbf{E}+02$ & $\#$ Fu_3 \\
1006 & $5.00000000 \mathbf{E}+03$ & $\# \mathrm{Fu} \_4$ \\
25 & $1.03211827 \mathbf{E}+02$ & $\#$ hh_1 \\
35 & $2.23656366 \mathbf{E}+03$ & $\#$ hh_2 \\
1035 & $4.50404752 \mathbf{E}+03$ & $\#$ hh_3 \\
37 & $2.30145102 \mathbf{E}+03$ & $\#$ Hpm_3 \\
39 & $5.10386526 \mathbf{E}+03$ & $\#$ Hpm_4 \\
24 & $8.03497269 \mathbf{E}+01$ & $\# \mathrm{VWp}$ \\
224 & $2.34618648 \mathbf{E}+03$ & $\# \mathrm{VXp}$ \\
23 & $9.11887000 \mathbf{E}+01$ & $\# \mathrm{VZ}$ \\
2023 & $3.85889765 \mathbf{E}+03$ & $\# \mathrm{VZp}$
\end{tabular}

donde $\mathrm{Fu}_{-} \mathrm{i}$ y $\mathrm{Fd}_{-} \mathrm{i}$ representan los estados de los cuatro quarks Up y los cinco quarks Down, respectivamente.

Todo lo anterior demuestra que el modelo $3-3-1$ con tres tripletes funciona correctamente generando las masas de los quarks.

\section{Conclusiones}

Hemos mostrado que el paquete SARAH del programa MATHEMATICA genera correctamente las matrices de masa analíticas para el modelo 3-3-1 con neutrinos derechos, para el caso con tres tripletes escalares, lo cual muestra la gran herramienta computacional con la que contamos en física de altas energías. SARAH permite calcular no solo masas fermionicas, sino también para los bosones de Gauge, análisis del sector del Higgs, corrientes etc. Con ello, se reduce el tiempo de realización de cálculos analíticos, a nivel de árbol y a mayor orden perturbativo. Luego, usando SPheno, que es un programa complemetario para realizar cálculos numéricos, hemos obtenido las masas de los quarks del modelo para mostrar que estas herramientas computacionales funcionan.

Tener tres tripletes escalares genera adecuadamente todas las masas de los quarks a nivel árbol, para este modelo, y con lo cual podemos concluir que al menos, en la generación de masas de los quarks es un modelo realista.

\section{Apéndice}

\section{A. Modelo 331 con tres tripletes de Higgs en SARAH}

A continuación mostramos los cuatro archivos que se requieren para ejecutar SARAH y generar el módulo de SPheno para el modelo con tres tripletes de Higgs. Por defecto, el programa SPheno siempre escribe en el espectro de masas de salida los valores conocidos experimentalmente de las masas de los quarks del Modelo Estándar. Para evitar esto se desactivaron las lineas 802-805 del archivo LoopMasses_331v4.f90 de nuestro módulo de SPheno, esto con el propósito de que SPheno calcule las respectivas masas de acuerdo con los parámetros propios del modelo.

\section{A.1 Archivo 331-v3.m}

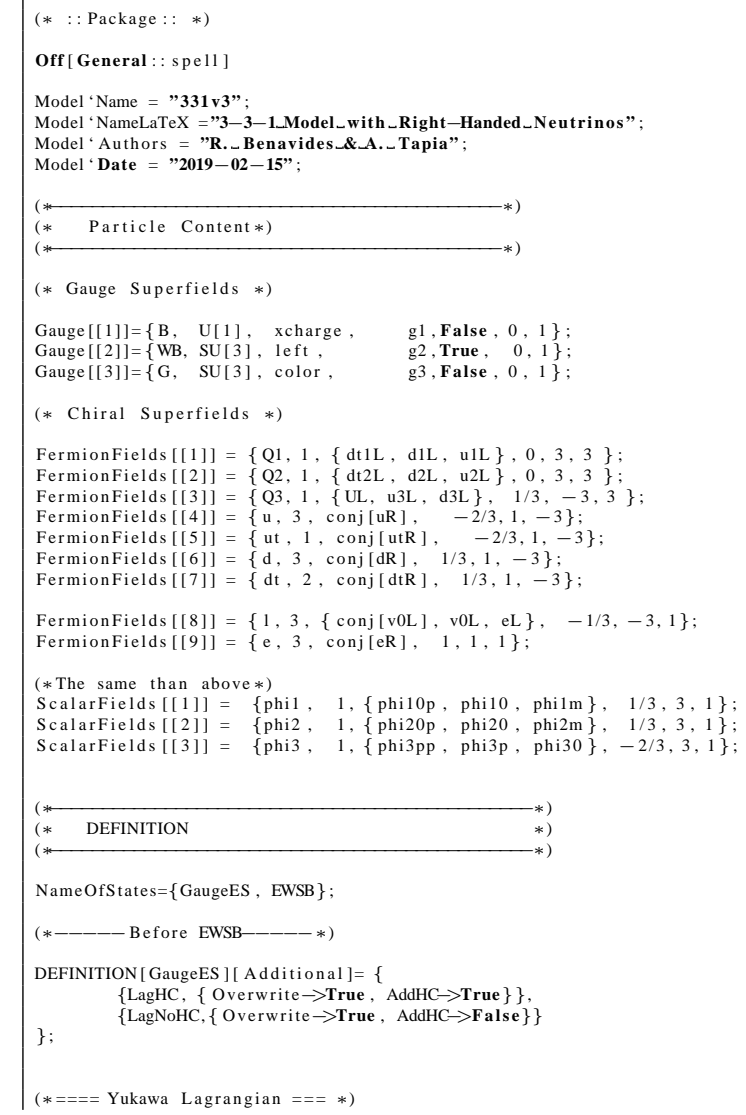




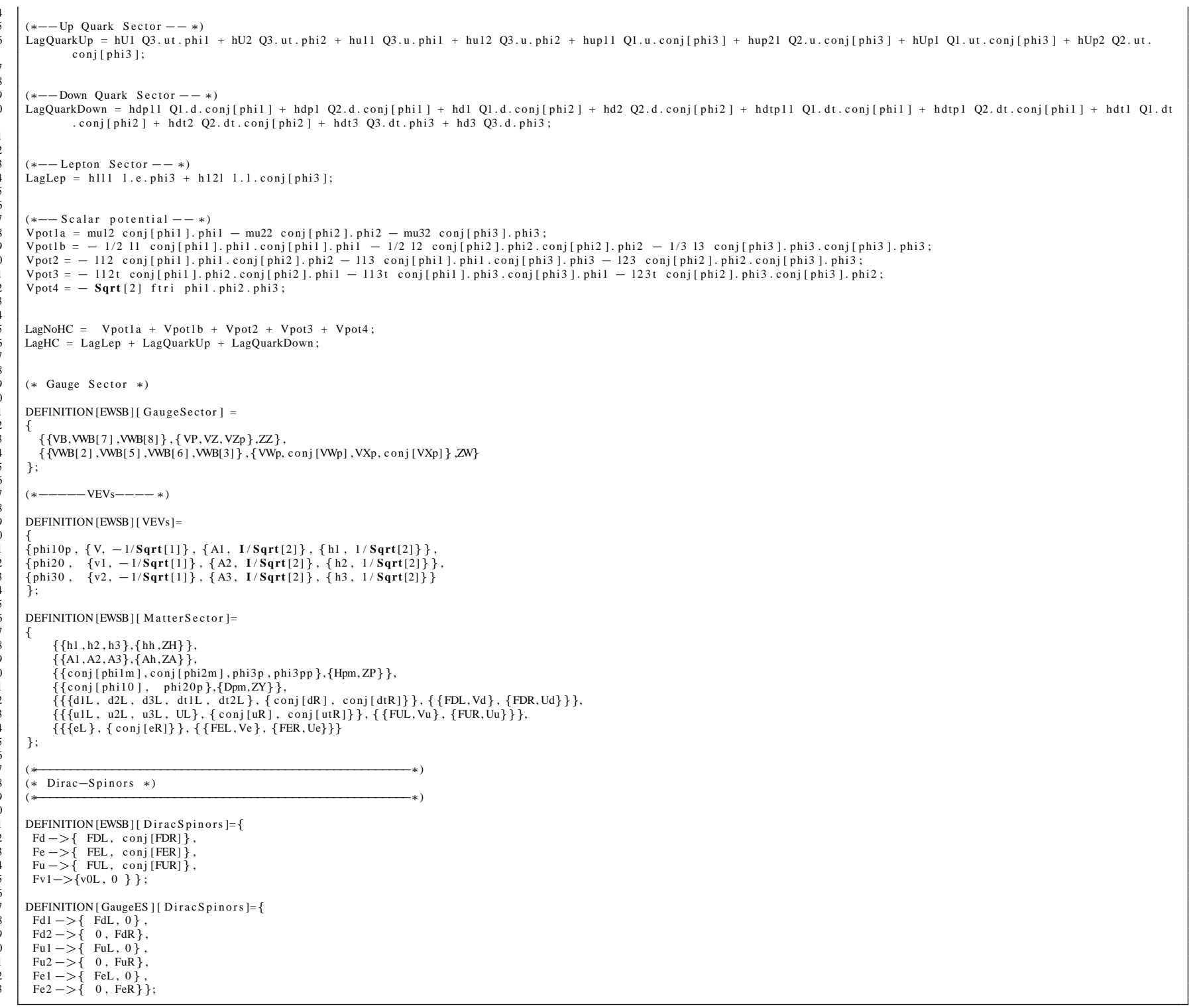

\section{A.2 Archivo parameters.m}

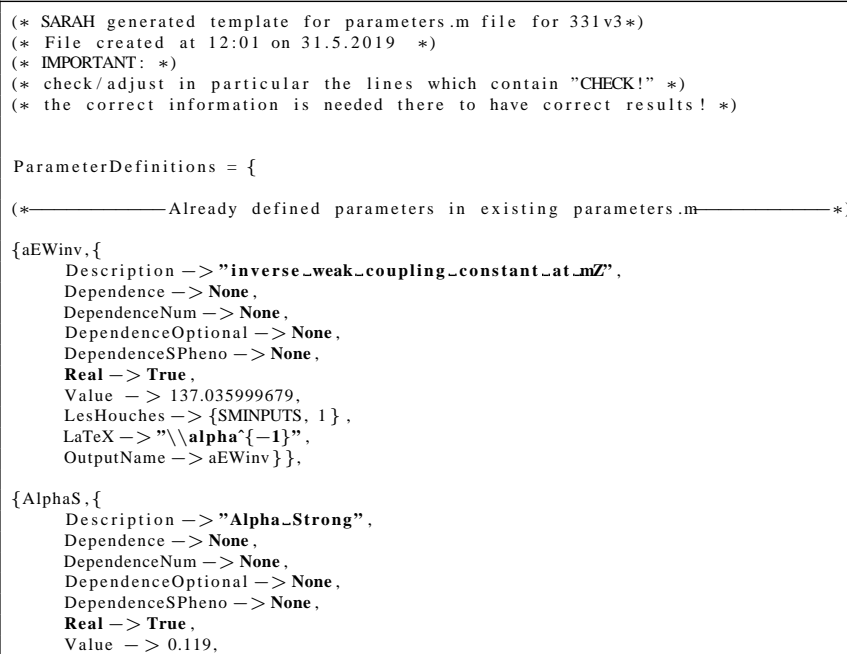


LesHouches $->$ \{SMINPUTS, 3$\}$,

LaTeX $->$ "\\alpha_S",

$\{\mathrm{e},\{$

Description $->$ "electric _charge"

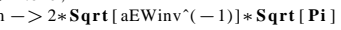

DependenceOptional $->$ None

Real $->$ True.

OutputName $->$ el $\}\}$,

$\{$ ftri, \{

Dependence $->$ None,

DependenceNum $\rightarrow>$ None,

DependenceOptional $->$ None,

Real $->$ True,

LesHouches $\rightarrow$ \{ POTENTIAL331, 5$\}$,

LaTeX $->$ "\} \backslash \text { epsilon" }

OutputName $->$ ftri $\}$

$\{\mathrm{g} 1,\{$

Description $->$ "X-Coupling",

Dependence $->$ None,

Dependencentione

Dependence

Real $\rightarrow$ True,

LesHouches $\rightarrow$ \{gauge, 1$\}$

LaTeX $\rightarrow$ " $\rightarrow$,

OutputName $->\mathrm{g} 1\}$ \},

$\{\mathrm{g} 2,\{$

Description -> "Left-Coupling"

Dependence $->$ None

DependenceNum $->\mathrm{e} *$ Csc [ ThetaW]

DependenceOptional $->\mathrm{e} * \operatorname{Csc}[$ ThetaW],

DependenceSPheno $->$ None,

Real $->$ True,

LesHouches $->\{$ gauge , 2$\}$,

LaTeX $->$ "g_2",
OutputName $->$ g2 $\}$

$\{\mathrm{g} 3,\{$

Description-> "Strong-Coupling",

Dependence $->$ None,

Dependis

DependenceOptional $->$ None

DependenceSPh

Real $->$ True,

LesHouches $\rightarrow>$,

OutputName $->$ g3 $\}$ \},

$\{\mathrm{Gf},\{$

Description $->$ "Fermi, s_constant",

Dependence $->$ None,

DependenceNum $->$ None,

DependenceOptional $->$ None

DependenceSPheno $->$ None,

Real $->$ True

Value $->0.0000116639$

LesHouches $\rightarrow$ \{SMINPUTS, 2$\}$,

LaTeX -> "G_f",

OutputName $->$ Gf $\}$ \},

$\{\mathrm{h} 121,\{$

Dependence $->$ None,

DependenceNum $->$ None

DependenceOptional $->$ None

DependenceSPh

Real $->$ False ,

LesHouches $->$ h121,

OutputName ->h121\}\},

\{hd $3,\{$

Dependence $->$ None,

DependenceOptional $->$ None,

DependenceSPheno $->$ None

Real $->$ False

LesHouches $->$ hd 3

LaTeX $->$ "h^ $\{\mathbf{d}\}-\{\mathbf{3}\}$,
OutputName $->$ hd 3$\}\}$,

$\{$ hdp $11,\{$

Dependence $->$ None,

DependenceNum $->$ None,

DependenceOptional $->$ None

DependenceSPheno $->$ None

Real $->$ False

LesHouches $->$ hdp 11 ,

LaTeX $->$ "h^ $\left\{\mathbf{d}^{\prime}\right\}-\{11\} "$,
OutputName $->$ hdp 11$\}\}$,

\{hd $1,\{$

\{
Dependence $->$ None,
DependenceNum $->$ None

DependenceOptional $->$ None

DependenceSPheno $\rightarrow>$ None,

Real $->$ False

LesHouches $\rightarrow$ hd 1

$\mathrm{LaTeX}->$ "h^ $\{\mathbf{d}\}-\{\mathbf{1}\}$ ",

OutputName $->$ hd 1$\}$,

$\{$ hdp $1,\{$

Dependence $->$ None, 
DependenceNum $\rightarrow>$ None

DependenceOptional $->$ None DependenceSPheno $\rightarrow>$ None, Real $->$ False ,

LesHouches $\rightarrow>$ hdp 1,
LaTeX $\rightarrow$ h^ $\{$ d' $\}$,

LaTeX $->{ }^{\prime}{ }^{\wedge}\left\{\mathbf{d}^{\prime}\right\}-\{\mathbf{1}\}$,
OutputName $->$ hdp1 $\}$

\{hd2, \{

Dependence $->$ None,

DependenceOptional $\rightarrow>$ None

DependenceSPheno $->$ None,

Real $->$ False

LesHouches $\rightarrow>$ hd 2 ,

LaTeX $->$ "h^ $\{\mathbf{d}\}-\{\mathbf{2}\},$,
OutputName $->$ hd 2$\}\}$,

$\{$ hdt $3,\{$

Dependence $->$ None,

DependenceNum $->$ None

DependenceOptional $->$ None

DependenceSPheno $->$ None,

Real $->$ False,

LesHouches $->$ hdt 3 ,

LaTeX $->$ "h^ $\{\mathbf{D}\}-\{\mathbf{3}\} "$,
OutputName $->$ hdt 3$\}\}$,

\{hdtp11, \{

Dependence $->$ None,

DependenceNum $->$ None,

DependenceOptional $->$ None

Real $->$ False

LesHouches $->$ hdtp 11 .

LaTeX $->$ "h^ $\left\{\mathbf{D}^{\prime}\right\}-\{11\}$ ",

OutputName $->$ hdtp11\}\},

$\{$ hdt $1,\{$

Dependence $->$ None,
DependenceNum $\rightarrow>$ None,

DependenceOptional $\rightarrow$ None

DependenceSPheno $->$ None,

Real $->$ False

LesHouches $->$ hdt 1 ,

LaTeX $->$ "h^ $\{\mathbf{D}\}-\{\mathbf{1}\} "$,
OutputName $->$ hdt 1$\}\}$,

$\{$ hdtp $1,\{$

Dependence $->$ None

DependenceOptional $\rightarrow>$ None,

DependenceOptional $->$ None
DependenceSPheno $->$ None,

Real $->$ False

LesHouches $->$ hdtp 1

LaTeX $\rightarrow$ " $^{\wedge}\left\{D^{\prime}\right\}-\{1\}$,

OutputName $->$ hdtp1\}\},

$\{$ hdt $2,\{$

Dependence $->$ None,

DependenceOptional $\rightarrow$ None,

DependenceSPheno $->$ None

Real $->$ False

LesHouches $\rightarrow>$ hdt2,

LaTeX $->$ "h^\{D\}-\{2\}",
OutputName $->$ hdt 2$\}\}$,

$\{\mathrm{h} 111,\{$

Dependence $->$ None

DependenceNum $\rightarrow>$ None,

DependenceOptional $->$ None, DependenceSPheno $->$ Non

Real $->$ False,

LesHouches $->$ hll1

OutputName $->$ h11 $\}\}$,

$\{\mathrm{hU} 1,\{$

Dependence $->$ None,

DependenceOptional $\rightarrow$ None

DependenceSPheno $->$ None

Real $->$ False,

LesHouches $->$ hU1

LaTeX $->$ "h`U_.1",

OutputName $->$ hU1 $\}$ \},

$\{$ hu11, \{

Dependence $->$ None,

DependenceNum -> None,

DependenceOptional $->$ None,

DependenceSPheno $->$ None,

Real $->$ False,

LesHouches $->$ hu11,

$\left.\begin{array}{l}\text { LaTeX }->\text { "h⿱ } \mathbf{u}_{-}\{\mathbf{1 1}\} ", \\ \text { OutputName }->\text { hu11 }\}\end{array}\right\}$,

\{hu12, \{

Dependence $->$ None,

Dependence $>>$ None,

DependenceSPheno $\rightarrow$ None,

Real $->$ False

LesHouches $\rightarrow>$ hu 12 .

LaTeX $->$ "h⿱ $\mathbf{u}_{-}\{\mathbf{1 2}\}$ ",

OutputName $->$ h12 $\}$ \},

$\{\mathrm{hU} 2,\{$

Dependence $->$ None,
DependenceNum $->$ None

Rev. Mex. Fís. E 18 (1) 107-126 

DependenceSPheno $->$ None, Real $->$ False

LesHouches $\rightarrow>$ hU2

OutputName $->$ hU2 $\}$ \},

$\{\mathrm{hUp} 1$,

Dependence $->$ None

DependenceNum $\rightarrow>$ None,

DependenceSPheno $->$ None,

Real $->$ False ,

LesHouches $\rightarrow$ hup1,

LaTeX $\rightarrow>$ "h^ $\left\{\mathbf{U}^{\prime}\right\}-\{\mathbf{1}\}$,

\{hup $11,\{$

Dependence $->$ None,
DependenceNum $\rightarrow$ None

DependenceNum $\rightarrow>$ None

DependenceOptional $->$ None

DependenceSPheno $->$ None,

Real $->$ False

LesHouches $->$ hup 11 ,

LaTeX $->{ }^{\prime}$ ' $\left\{\mathbf{u}^{\prime}\right\}-\{\mathbf{1 1}\}$
OutputName $->$ hup 11$\}\}$,

$\{\mathrm{hUp} 2$,

Dependence $->$ None,

DependenceNum $->$ None,

DependenceOptional $->$ None

DependenceSPh

LesHouches $\rightarrow$ hUp2

LaTeX $->$ "h^ $\left\{\mathbf{U}^{\prime}\right\}-\{2\}$,

OutputName $->$ hUp 2$\}\}$,

$\{$ hup $21,\{$

Dependence $->$ None,

DependenceNum $\rightarrow>$ None,

DependenceOptional $->$ None

DependenceSPheno

Real $->$ False

LesHouches $\rightarrow>$ hup 21,

LaTeX $->$ "h $\left\{\mathbf{u}^{\prime}\right\}_{-}\{\mathbf{2 1}\}$ ",

OutputName $->$ hup 21$\}\}$,

$\{11,\{$

Dependence $->$ None,

DependenceNum $\rightarrow>$ None,

Dependenceptional $\rightarrow$ None,

DependenceSPhe

LesHouches $->$ \{POTENTIAL331, 6$\}$,

LaTeX - > \\lambda $\{1\}$,

OutputName $->11\}\}$.

$\{112$

Dependence $->$ None,

DependenceOptional $->$ None

DependenceSPheno $->$ None,

Real $->$ False ,

LesHouches $->$ PPOTENTIAL331, 11 $\}$

LaTeX $->$ " \\lambda $\{\mathbf{1 2}\}$ ",

OutputName $->112\}\}$

$\{112 \mathrm{t}$,

Dependence $->$ None,

DependenceNum $\rightarrow>$ None

DependenceOptional $->$ None

DependenceSPheno $->$ None

LesHouches $->$ \{POTENTIAL331, 16\},

LaTeX $->$ Outilide $-\backslash \backslash$ lambda $\{12\}$,

$\{113,\{$

Dependence $->$ None,

Dependence $>$ None,

Dependencespheno $\rightarrow$ None.

Real $->$ False ,

LesHouches $->$ \{POTENTIAL331, 12\}

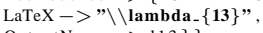

OutputName $->113\}\}$.

$\{113 \mathrm{t},\{$

Dependence $->$ None,

DependenceNum $->$ None

DependenceOptional $->$ None

DependenceSPheno $->$ None

LesHouches $\rightarrow$ \{POTENTIAL331, 15 $\}$,

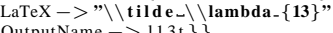

OutputName $->113 \mathrm{t}\}$ \},

$\{12$,

Dependence $->$ None,

DependenceOptional $\rightarrow$ None,

DependenceSPhenal $>$ None,

Real $\rightarrow>$ False

LesHouches $\rightarrow$ \{ POTENTIAL 331, 7\}

LaTeX $->$ "\lambda $\{$ 2 $\}$ ",

OutputName $->12\}\}$,

$\{123,\{$

Dependence $->$ None,

DependenceNum $->$ None,
DependenceOptional $->$ None 


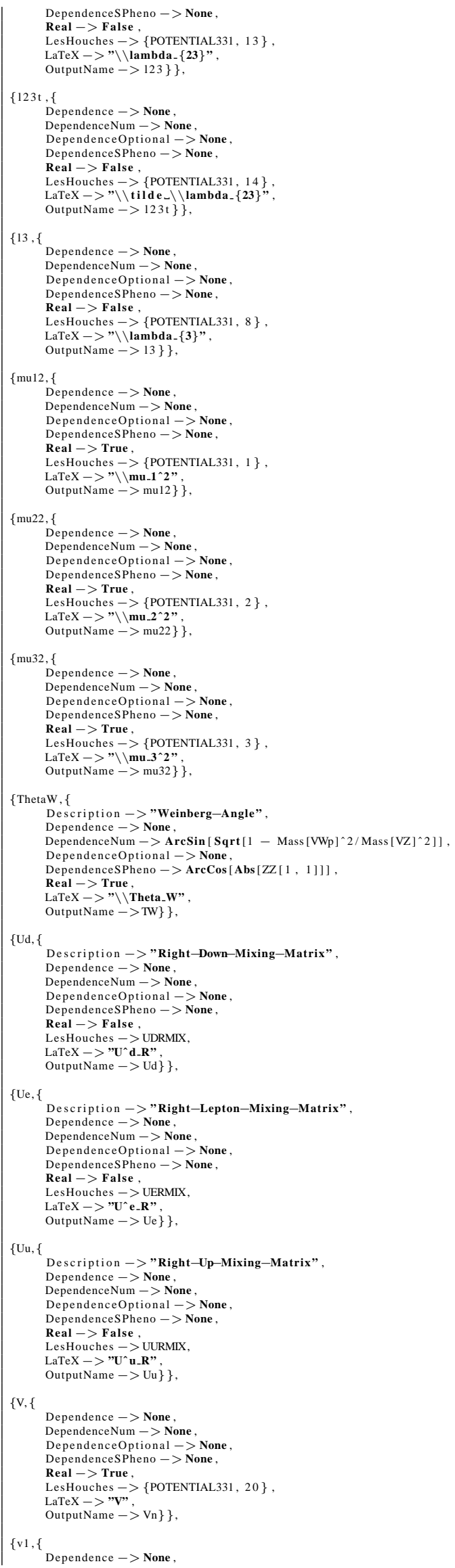


DependenceNum $->$ None,

DependenceOptional $->$ None

DependenceSPheno $->$ None

Real $->$ True

LesHouches $\rightarrow$ PPOTENTIAL331, 18$\}$

OutputName $->\mathrm{v} 1\}\}$,

$\{\mathrm{v} 2,\{$

Dependence $->$ None

DependenceNam $->$ None

DependenceSPheno $\rightarrow>$ None

Real $->$ True,

LesHouches $\rightarrow$ POTENTIAL331, 19$\}$

LaTeX $->$ "v $\{\mathbf{2}\}$ ",

$\{\mathrm{Vd}$,

Description-> "Left-Down-Mixing-Matrix",

Dependence $->$ None,

DependenceNum $->$ None

DependenceOptional $->$ None

DependenceSPheno $\rightarrow>$ None

Real $->$ False

LesHouches $->$ UDLMIX

LaTeX $->$ "UA_L"
OutputName $->$ Vd $\}$

$\{\mathrm{Ve}$,

Description-> "Left-Lepton-Mixing-Matrix"

Dependence $\rightarrow$ None

enceNum $->$ None

DependenceOptional $\rightarrow>$ None

Real $->$ False

LesHouches $->$ UELMIX

LaTeX $\rightarrow>$ "U^e_L",

$\{\mathrm{Vu}$,

Description $->$ "Left-Up-Mixing-Matrix",

Dependence $->$ None,

DependenceNum $->$ None

DependenceOptional $\rightarrow>$ None

DependenceSPheno $->$ None,

Real $->$ False,

LesHouches $->$ UULMIX

LaTeX $\rightarrow>$ "U u_L",

$\{$ ZA, $\{$ Description $->$ "Pseudo-Scalar-Mixing-Matrix"

Dependence $\rightarrow$ None

DependenceNum $\rightarrow$ No

DependenceOptional $\rightarrow$ None,

DependenceSPheno $\rightarrow$ None,

Real $->$ False

LesHouches $->$ ZAMIX

LaTeX $\rightarrow>\mathbf{Z}^{\wedge}\{\mathbf{A}\}$, ,

OutputName $->$ ZA $\}$

$\{\mathrm{ZH}$,

Description -> "Scalar-Mixing-Matrix"

Dependence $->$ None,

DependenceNum $->$ None

DependenceOptional $->$ None

DependenceSPheno $->$ None,

Real $->$ False

LesHouches $->$ ZHMIX

$\mathrm{LaTeX}->\mathbf{Z}^{\wedge}\{\mathbf{H}\} "$

OutputName $->\mathrm{ZH}\}$,

$\{\mathrm{ZP},\{$

Description -> "Charged-Mixing-Matrix"

Dependence $->$ None,

DependenceOptional $\rightarrow$ None,

Dependencespheno $\rightarrow$ None

Real $\rightarrow$ False

LesHouches $\rightarrow$ ZPMIX,

LaTeX $\rightarrow>$ "Z $\{\backslash \backslash \mathbf{p m}\}$ ",

OutputName $->$ ZP $\}$.

$\{\mathrm{ZW}$,

Description $->$ "Charged_gauge_boson_Mixing_Matrix"

Dependence $->\{\{1 / \mathbf{S q r t}[2], 1 / \mathbf{S q r t}[2], 0,0\},\{(-\mathbf{I}) / \mathbf{S q r t}[2], \mathbf{I} / \mathbf{S q r t}[2], 0,0\},\{0,0,-(1 / \mathbf{S q r t}[2]),-(1 / \mathbf{S q r t}[2])\},\{0,0, \mathbf{I} / \mathbf{S q r t}[2],(-\mathbf{I}) / \mathbf{S q r t}[2]\}\}$,

DependenceNum $\rightarrow>$ None

DependenceOptional $\rightarrow>$ None

DependenceSPheno $->$ None

Real $\rightarrow>$ False

LesHouches $->$ ZWMIX

$\mathrm{LaTeX}->$ "Z⿱ $\{\mathbf{W}\}$ "

OutputName $->Z W\}\}$,

$\{\mathrm{ZY}$,

Dependence $->$ None,

Dependence $\rightarrow>$ None,

Dependencers

Real $->$ False,

Real $->$ False,
LesHouches $->$ ZY

LaTeX $->$ "Z_Y",

OutputName $->$ zy $\}$ \}

$\{\mathrm{ZZ}$,

Description -> Neutral_gauge_boson_Mixing_Matrix",

Dependence $->$ None,

DependenceNum $->$ None

DependenceOptional $->$ None
DependenceSPheno $->$ None, 


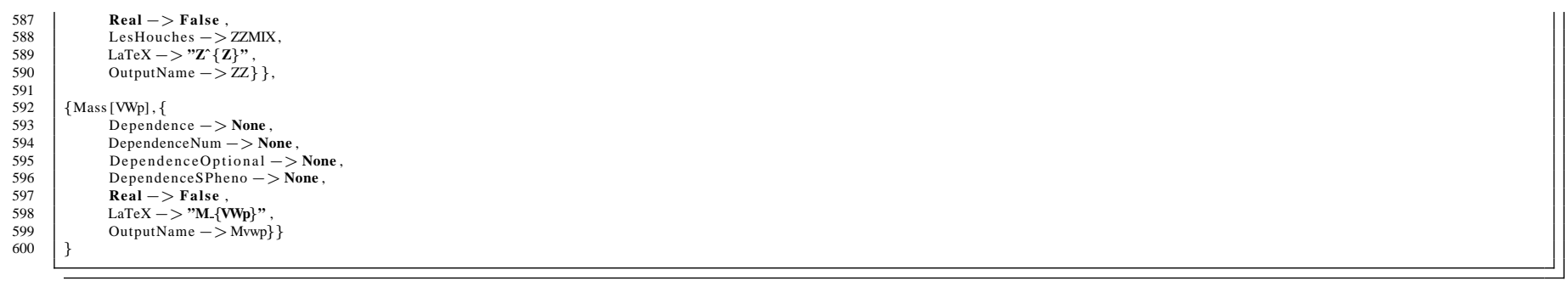

\section{A.3 Archivo particles.m}

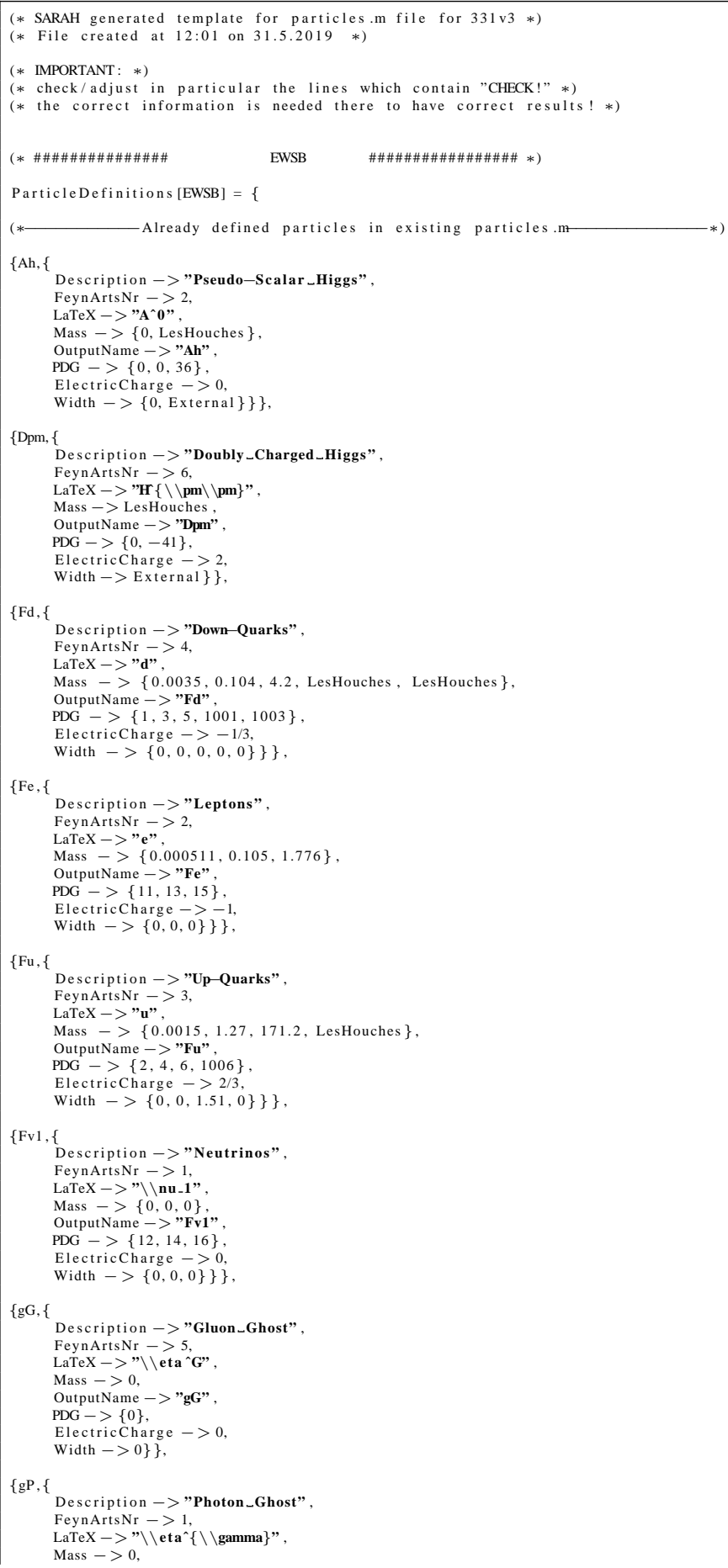




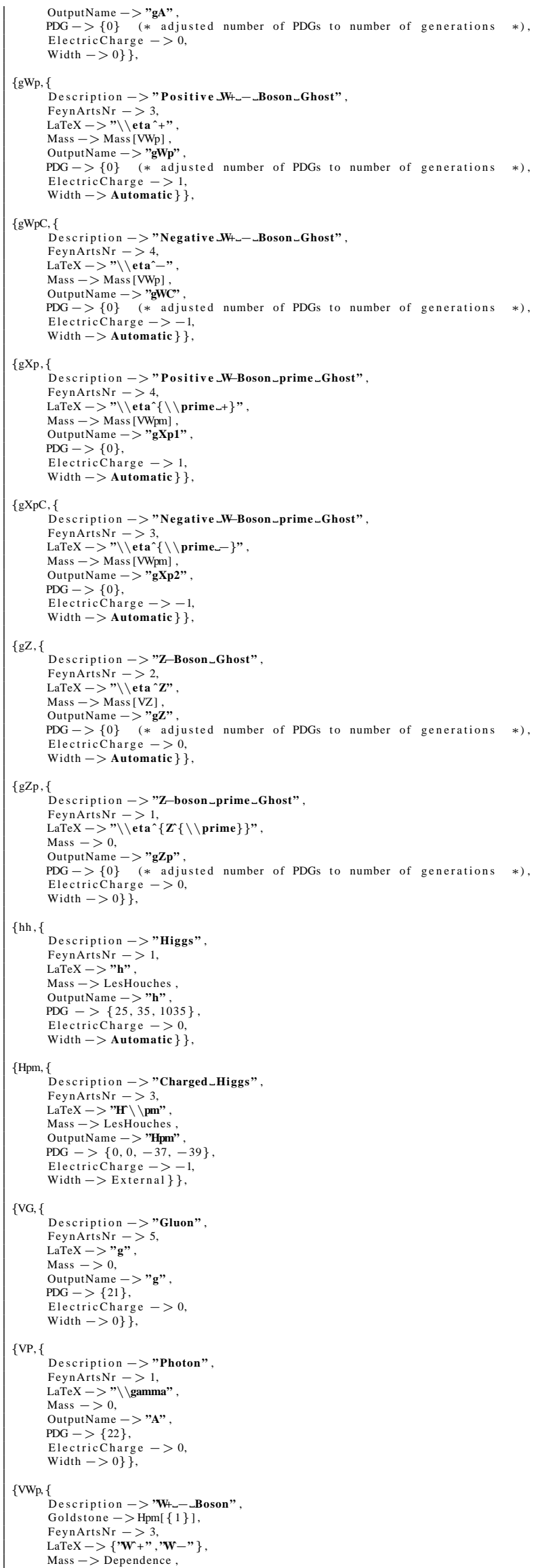




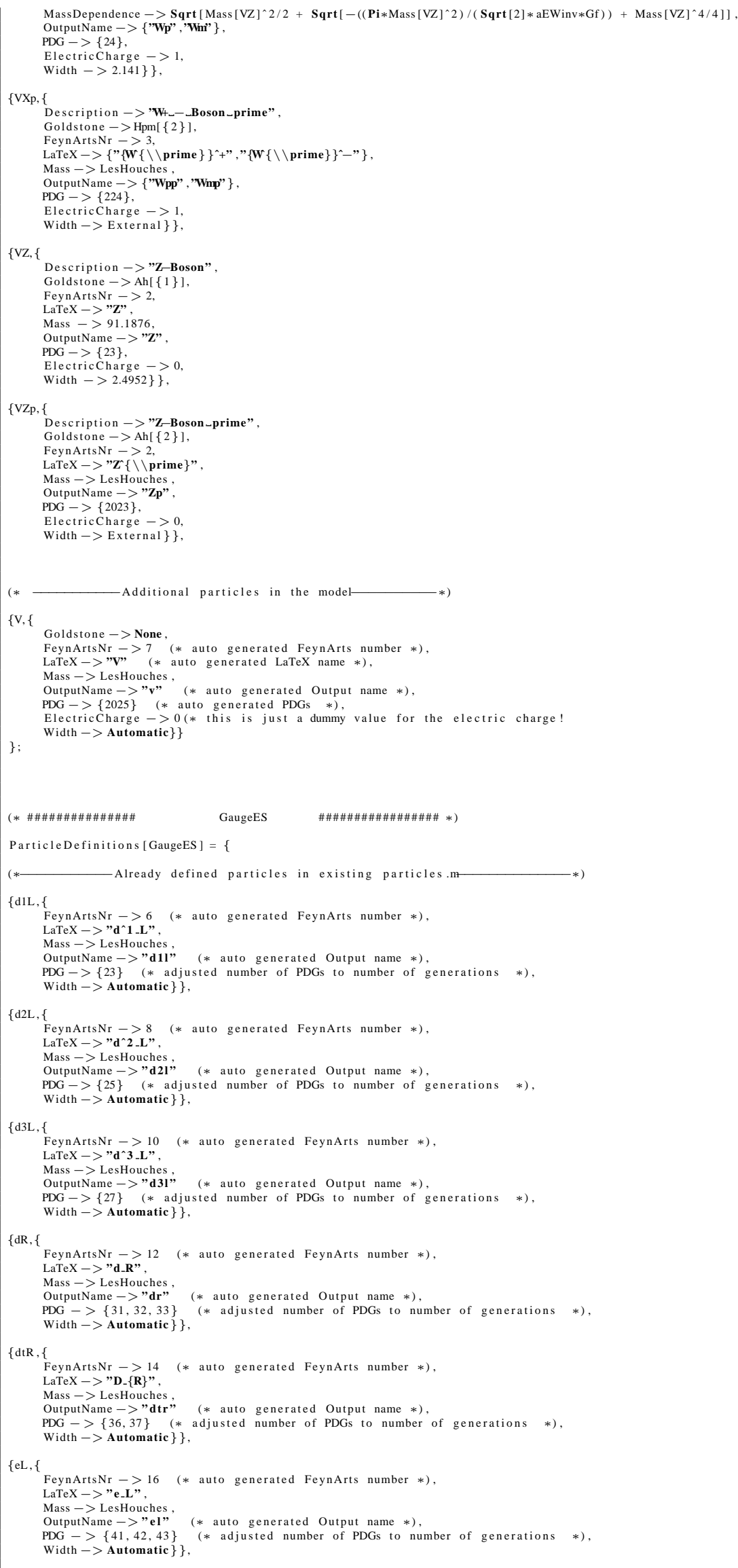




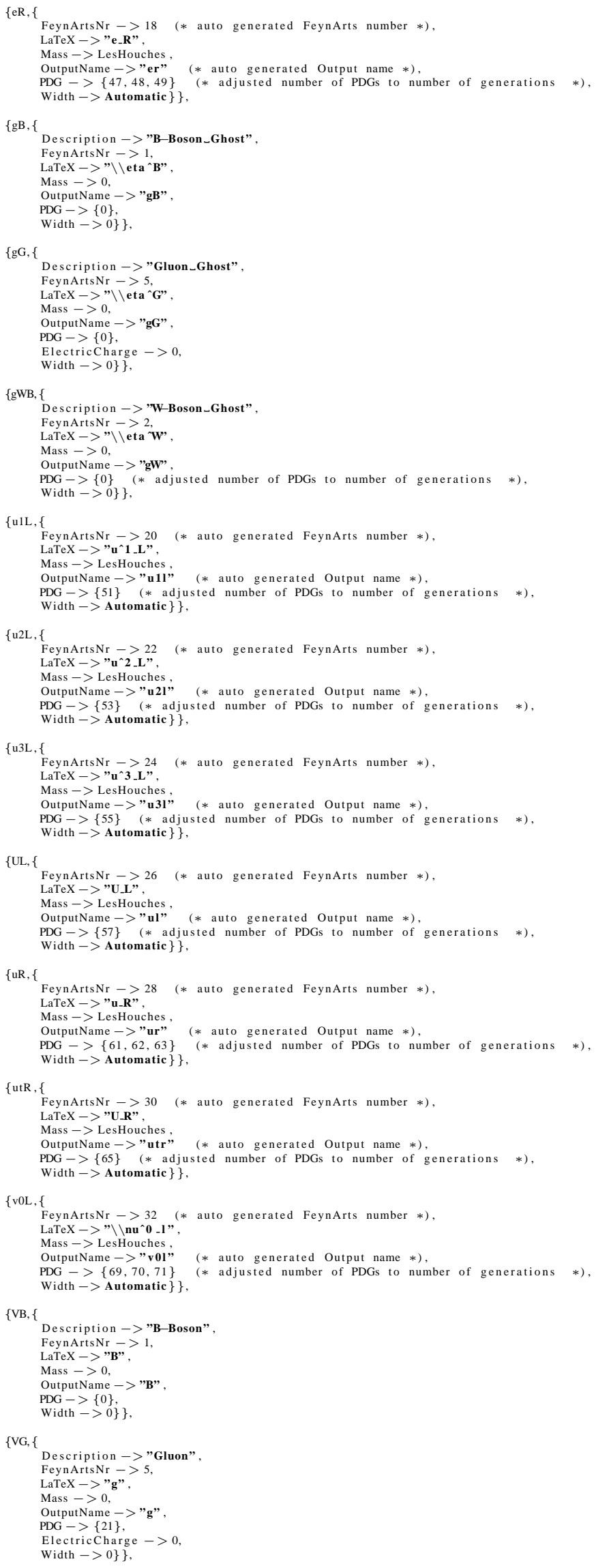




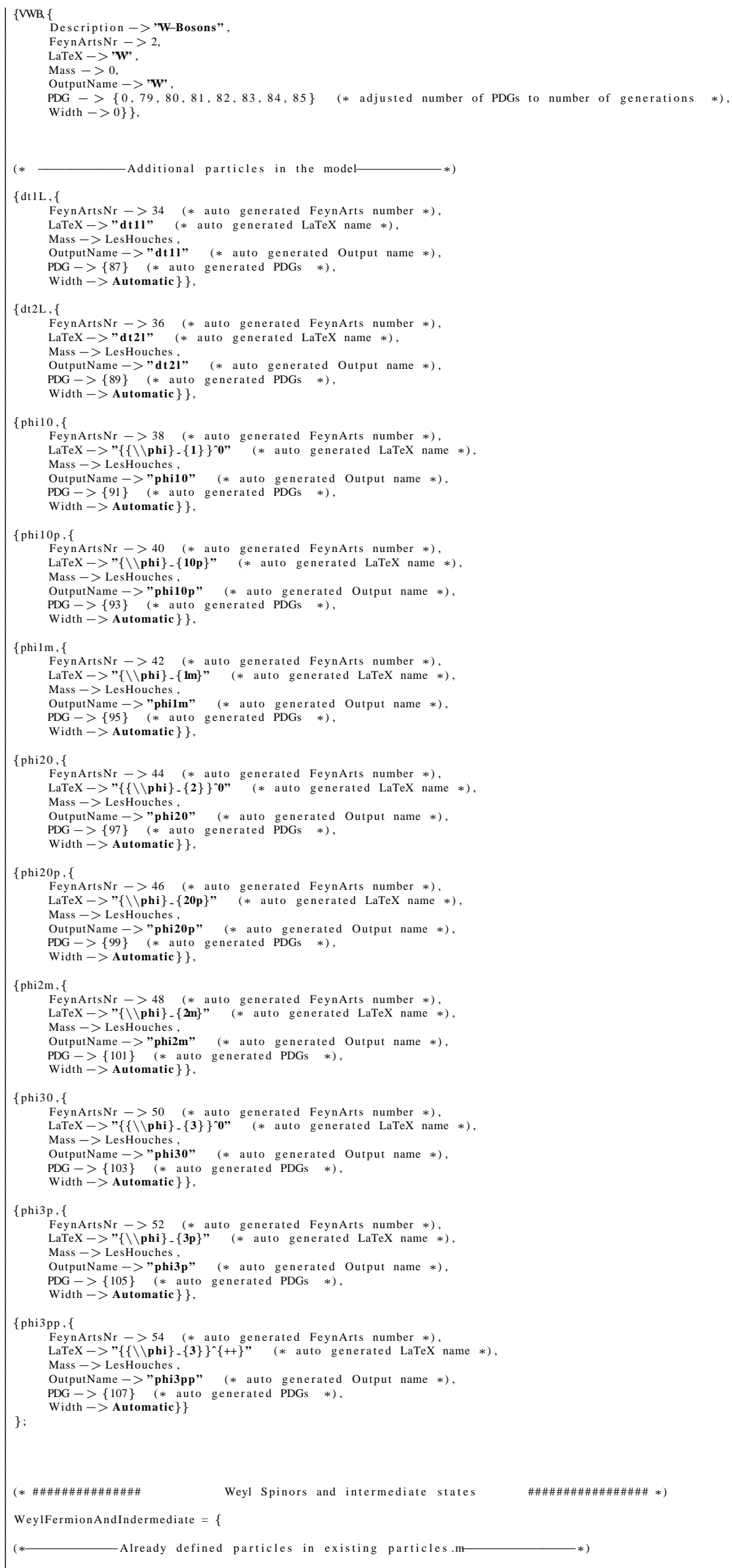




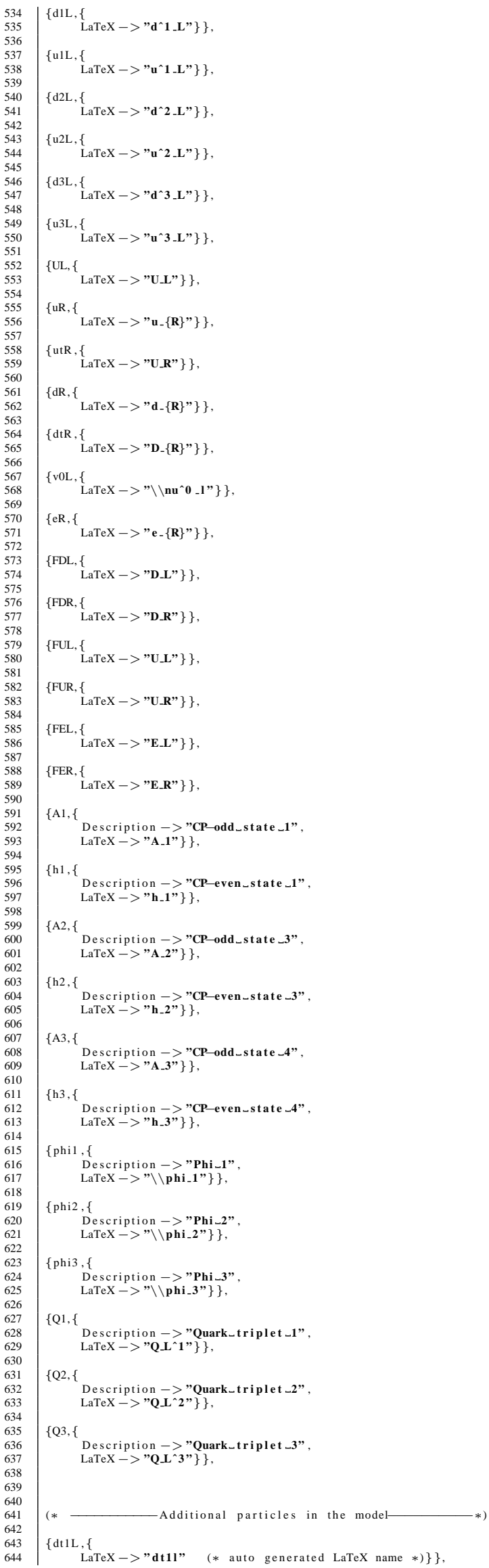




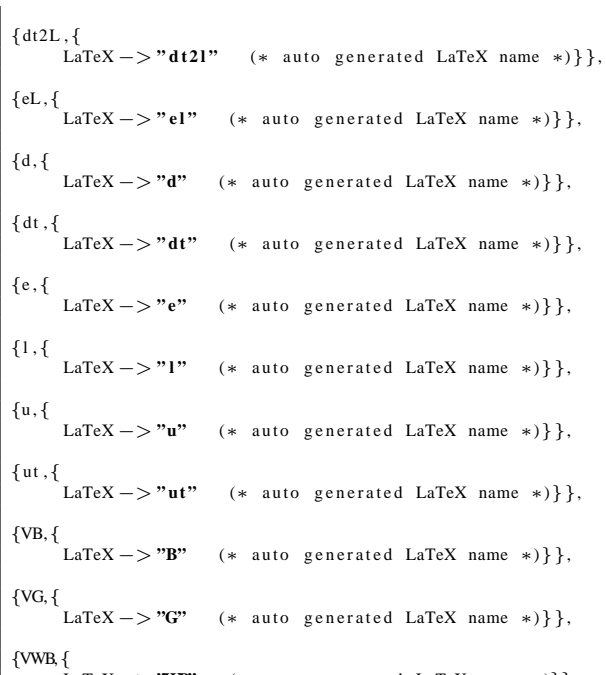

\section{A.4 Archivo SPheno.m}

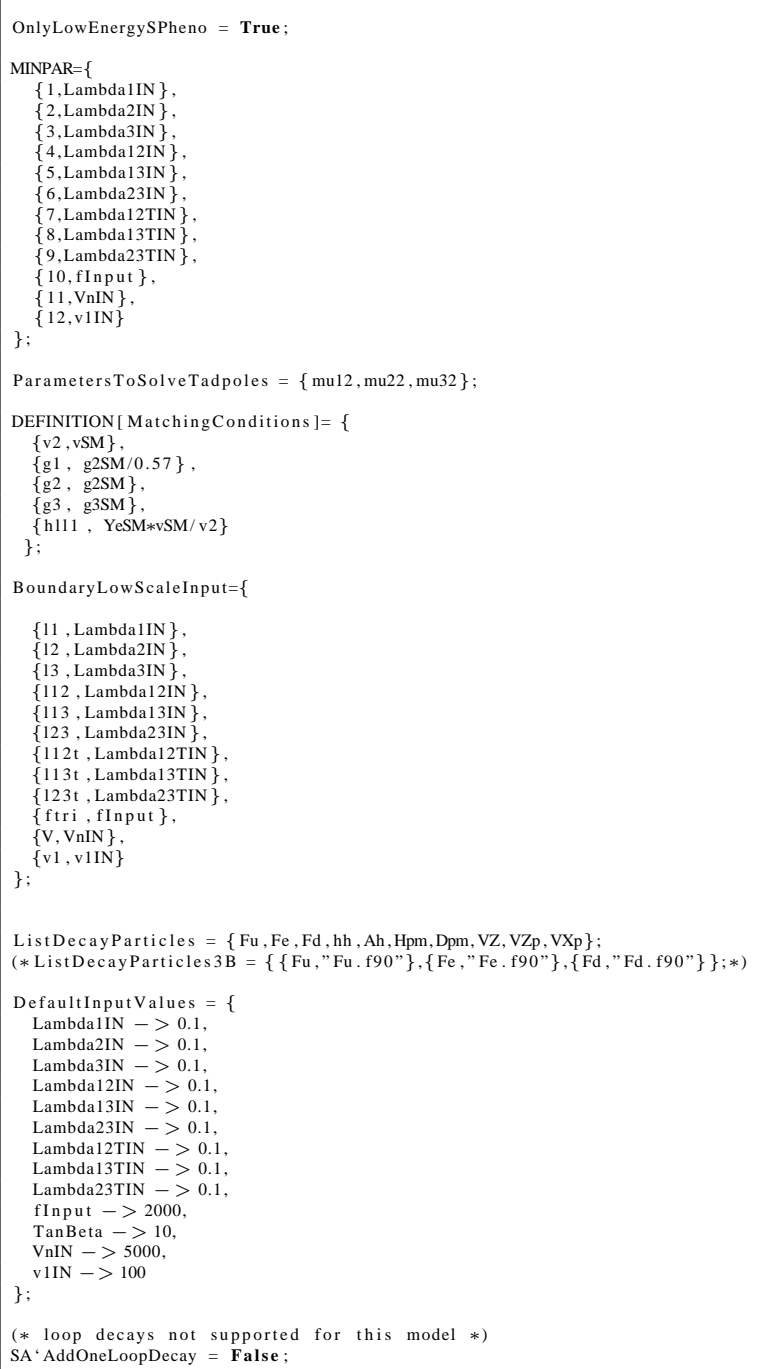




\section{Agradecimientos}

Agradecemos a Diego Restrepo de la Universidad de Antioquia por las útiles discusiones sobre SARAH. AT agradece el soporte financiero de la Universidad de Medellín a través del proyecto 1042. RHB agradece al 'Centro de Laboratorios de investigación, parque i-ITM'.
1. K. T. Mahanthappa and P. K. Mohapatra, Phys. Rev. D42 (1990) 1732.

2. R. Martinez and William A. Ponce and Luis A. Sanchez, Phys. Rev. D65 (2002) 055013, https://doi.org/10.1103/ PhysRevD.65.055013

3. Luis A. Sanchez and William A. Ponce and R. Martinez, Phys. Rev. D64 (2001) 075013, https://doi.org/10.1103/ PhysRevD.64.075013

4. H. Fanchiotti and C. Garcia-Canal and W. A. Ponce, Europhys. Lett. 72 (2005) 733-739, https://doi.org/10.1209/ epl/i2005-10313-x.

5. V. Pleitez and M. D. Tonasse, Phys. Rev. D48 (1993) 2353-2355, https://doi.org/10.1103/PhysRevD. 48.2353

6. William A. Ponce and Juan B. Florez and Luis A. Sanchez, Int. J. Mod. Phys. A17, (2002) 643-660, https://doi.org/ $10.1142 / \mathrm{S} 0217751 \times 02005815$.

7. F. Pisano and V. Pleitez, Phys. Rev. D46 (1992) 410-417, https://doi.org/10.1103/PhysRevD.46.410

8. P. H. Frampton, Phys. Rev. Lett. 69 (1992) 2889, https: //doi.org/10.1103/PhysRevLett.69.2889.

9. Murat Ozer, Phys. Rev. $D 54$ (1996) 4561, https://doi. org/10.1103/PhysRevD.54.4561.

10. R. D. Peccei and Helen R. Quinn, Phys. Rev. Lett. 38 (1977) 1440, https://doi.org/10.1103/PhysRevLett. 38.1440

11. Alex G. Dias and C. A. de S. Pires and P. S. Rodrigues da Silva, Phys. Rev. D68 (2003) 115009, https://doi.org/ 10.1103/PhysRevD.68.115009

12. Paul H. Frampton, arXiv:hep-ph/9507351 (1995).

13. Douglas Fregolente and Mauro D. Tonasse, Phys. Lett. B555 (2003) 7, https://doi.org/10.1016/ S0370-2693(03)00037-6

14. Hoang Ngoc Long and Nguyen Quynh Lan, Europhys. Lett. 64 (2003) 571, https://doi.org/10.1209/epl/ i2003-00267-5

15. Simonetta Filippi and William A. Ponce and Luis A. Sanchez, Europhys. Lett. 73 (2006) 142, https://doi.org/10. 1209/epl/i2005-10349-x
16. Yutaka Okamoto and Masaki Yasue, Phys. Lett. B466 (1999) 267, https://doi.org/10.1016/S0370-2693(99) $01134-\mathrm{X}$

17. Teruyuki Kitabayashi and Masaki Yasue, Phys. Rev. D63 (2001) 095002, https://doi.org/10.1103/ PhysRevD.63.095002.

18. Darwin Chang and Hoang Ngoc Long, Phys. Rev. D73 (2006) 053006, https://doi.org/10.1103/PhysRevD. 73 . 053006 .

19. Aharon Davidson and Kameshwar C. Wali, Phys. Rev. Lett. 59 (1987) 393, https://doi.org/10.1103/ PhysRevLett.59.393.

20. S. Rajpoot, Phys. Rev. D36 (1987) 1479, https://doi. org/10.1103/PhysRevD.36.1479.

21. Darwin Chang and Rabindra N. Mohapatra, Phys. Rev. Lett. 58 (1987) 1600, https://doi.org/10.1103/ PhysRevLett.58.1600

22. D. A. Gutierrez and W. A. Ponce and L. A. Sánchez, Int. J. Mod. Phys. A21 (2006) 2217, https://doi.org/10. $1142 / \mathrm{S} 0217751 \times 06029442$

23. Benavides, Richard H. and Giraldo, Yithsbey and Ponce, William A., Phys. Rev. D80 (2009) 113009, https: / / doi. org/10.1103/PhysRevD.80.113009

24. F. Staub, arXiv:hep-ph/0806.0538 (2008).

25. Vicente, Avelino, arXiv:hep-ph/1507.06349 (2015).

26. Wolfram Research, Inc.Mathematica, Version 11.1, Campaign I, (2018).

27. Werner Porod, Comput. Phys. Commun. 153 (2003) 275, https://doi.org/10.1016/s0010-4655(03) $00222-4$

28. Porod, W. and Staub, F.", Comput. Phys. Commun. 183 (2012) 2458, https://doi.org/10.1016/j.cpc.2012. 05.021 .

29. P.A. Zyla et al, to be published in Prog. Theor. Exp. Phys. 083C01 (2020), http: //pdg.lbl.gov 